\title{
01
}

\section{Влияние потери данных на диагностику сложных режимов динамики}

\author{
(C) О.Н. Павлова ${ }^{1}$, А.Н. Павлов ${ }^{1,2, q}$ \\ ${ }^{1}$ Саратовский национальный исследовательский государственный \\ университет им. Н.Г. Чернышевского, Саратов, Россия \\ ${ }^{2}$ Саратовский государственный технический университет \\ им. Гагарина Ю.А., Саратов, Россия \\ ฯ E-mail: pavlov.alexeyn@gmail.com
}

\section{Поступило в Редакцию 10 апреля 2018 г.}

Обсуждается задача количественного описания динамики сложных систем по экспериментальным данным, содержащим сбойные участки, появляющиеся из-за артефактов, изменений условий функционирования или сбоев регистрирующего оборудования. Удаление таких участков может привести к изменению структуры сигналов, в связи с чем необходимо иметь представление о степени влияния данной процедуры на вычисляемые количественные характеристики. На примере многомасштабного анализа церебрального кровотока мы показываем, что даже экстремальная потеря данных сохраняет возможность правильной диагностики режима функционирования системы.

DOI: 10.21883/PJTF.2018.14.46348.17325

Сигналы, записанные в ходе экспериментальных исследований, часто содержат различные сбойные участки (артефакты), появление которых может быть связано как с анализируемым объектом, так и с регистрирующим оборудованием [1]. Наличие таких фрагментов данных усложняет анализ динамики исследуемой системы и может приводить к ошибочной диагностике режима ее функционирования. Характерным примером служат нейрофизиологические исследования, в которых различного рода артефакты присутствуют на участках, составляющих до 90\% записи электроэнцефалограммы [2,3]. Стандартным приемом очистки экспериментальных данных от помех является их фильтрация. За последние годы в этой области был предложен ряд новых решений, использующих, в частности, вейвлет-преобразование [4-11]. Соответствующие фильтры способны устранять локализованные помехи, удаление которых в рамках классических методов фильтрации является чрезвычайно сложной задачей [12-16]. Тем не менее этот прием далеко не 
всегда оказывается эффективным: если частотные диапазоны сигнала и помехи перекрываются, то фильтрация приводит к существенным искажениям сигнала. По этой причине широко используется подход, состоящий в удалении сбойных участков из экспериментальной записи, и последующий анализ проводится для сигнала, составленного из фрагментов, не содержащих артефактов. Если при этом исключается большое число сравнительно малых по длительности участков, то такая процедура может существенно влиять на корреляционные характеристики сигнала. В частности, в работах $[17,18]$ отмечалось, что потеря данных особенно сильно сказывается при анализе антикоррелированных процессов.

Тем не менее во многих областях науки и техники невозможно избежать появления артефактов, поэтому необходимо иметь представление о степени влияния эффектов потери данных на диагностику режима функционирования системы. Задача настоящей работы - установить возможность диагностики режима динамики в случае экстремальной потери данных, составляющей до 90\% от исходного объема выборки. Дополнительная сложность решаемой задачи связана с удалением большого числа сегментов малой длительности. С учетом того, что для экспериментальных данных часто характерна нестационарность, отражающая изменение характеристик системы во времени, в качестве инструмента исследования нами был выбран многомасштабный анализ, который относится к универсальным приемам цифровой обработки сигналов, применимым как в случае стационарных процессов, так и в случае динамики систем с меняющимися во времени характеристиками.

Многомасштабный (или кратномасштабный) анализ [4-6] предусматривает разложение сигнала с использованием набора зеркальных фильтров: низкочастотного, определяемого с помощью скейлингфункции $\varphi(t)$, и высокочастотного, который задается вейвлет-функцией $\psi(t)$. С помощью этих функций формируются базисы

$$
\varphi_{j, k}=2^{j / 2} \varphi\left(2^{j} t-k\right), \quad \psi_{j, k}=2^{j / 2} \psi\left(2^{j} t-k\right)
$$

и осуществляется разложение сигнала на одном из уровней разрешения $(m)$

$$
x(t)=\sum_{k} s_{m, k} \varphi_{m, k}+\sum_{j \geq m} \sum_{k} d_{j, k} \psi_{j, k}(t) .
$$

В зависимости от решаемой задачи выбор фильтров варьируется: выбираются число нулевых моментов вейвлет-функций, их регулярность и т.д. Во многих приложениях применяются ортонормированные вейвлеты семейства Добеши [4]. После разложения сигнала в выбранном

6 Письма в ЖТФ, 2018, том 44, вып. 14 
базисе (2) определяется набор коэффициентов $d_{j, k}$. Их статистический анализ на разных масштабах $j$ широко используется при решении многих задач диагностики состояния исследуемой системы $[5,19]$. В частности, информативной характеристикой является зависимость от масштаба стандартного отклонения вейвлет-коэффициентов

$$
\sigma(j, N)=\sqrt{\frac{1}{N} \sum_{k=0}^{N-1}\left[d_{j, k}-\left\langle d_{j, k}\right\rangle\right]^{2}},
$$

где $N$ - число вейвлет-коэффициентов на анализируемом масштабе $j$. Эта зависимость вычисляется для разных базисных функций, чтобы выявить наиболее значимые различия между диагностируемыми режимами динамики.

Рассмотрим, как будет влиять удаление участков экспериментальных данных на результаты их многомасштабного анализа. С этой целью нами был выбран нестационарный процесс, отражающий динамику церебральных сосудов головного мозга мыши [20]. Сигналы скорости церебрального кровотока регистрировались с помощью метода лазерной спекл-интерферометрии, обладающего возможностью отслеживания динамики кровотока в распределенной сети церебральных сосудов с последующим выбором области (например, крупной вены или артерии), внутри которой анализируются изменения скорости кровотока во времени. Детали экспериментальной процедуры регистрации сигналов подробно изложены в работе [20]. Пример спекл-изображения, позволяющего идентифицировать сосуды различного размера, приведен на рис. 1. Сигнал скорости кровотока регистрировался с частотой 40 изображений в секунду в течение $10 \mathrm{~min}$ и составлял 24000 отсчетов. Для удаления участков сигнала применялся алгоритм [17], предусматривающий исключение случайно расположенных фрагментов небольшой длительности (от 1 до 20 отсчетов) из исходных данных, при этом интервалы времени между исключаемыми участками также имели случайное (нормальное) распределение.

Чтобы количественно охарактеризовать изменения структуры сигнала при удалении его фрагментов, оценивалась величина относительной погрешности

$$
E=\frac{\Delta_{\sigma}}{\sigma_{0}} \cdot 100 \%,
$$

где $\sigma_{0}-$ стандартное отклонение вейвлет-коэффициентов исходного сигнала (не содержащего артефактов и без удаления фрагментов),

Письма в ЖТФ, 2018, том 44, вып. 14 


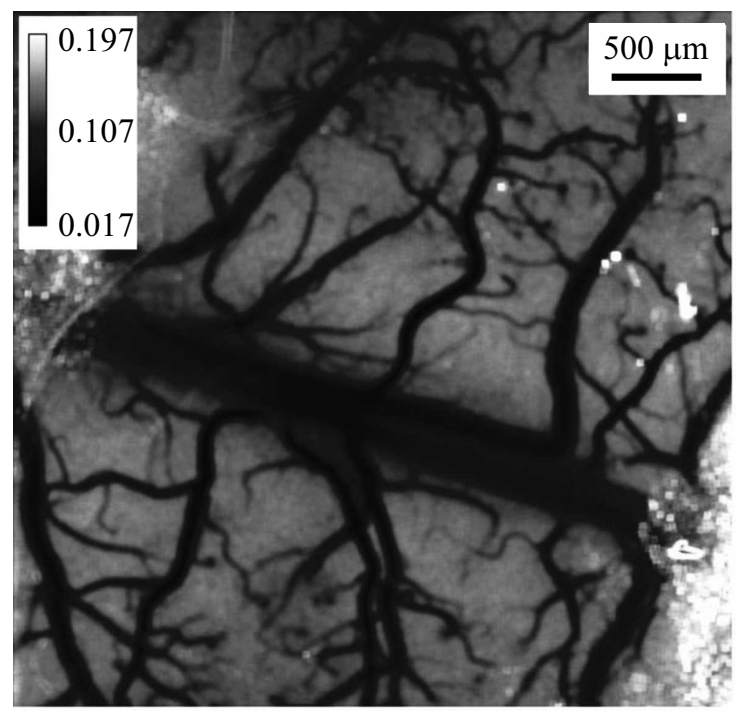

Рис. 1. Пример изображения, полученного при проведении спекл-интерферометрии, которое позволяет визуализировать церебральные сосуды лабораторной мыши и проводить оценку скорости церебрального кровотока в фиксированный момент времени. Тоновая шкала соответствует значениям скорости церебрального кровотока в нормированных единицах.

которое вычисляется на одном из масштабов $j$ (в соответствии с $[5,19]$ мы рассматривали $j=[4,5]$, но аналогичные результаты были получены и для других масштабов). Значение $\Delta_{\sigma}$ представляет собой абсолютную погрешность оценки величины $\sigma_{0}$ при рассмотрении сигнала с удаленными участками, которые в проводимом тестовом исследовании предполагаются сбойными. На рис. 2 показана зависимость величины $E$ от относительной длительности удаленных фрагментов $r$. Результаты приведены для значений $r$, меняющихся в диапазоне от 10\% (сравнительно небольшая потеря данных) до 90\% (экстремальная потеря данных). При этом изменение относительной погрешности $E$ составляет примерно от 1-2 до 7-8\%. Это позволяет сделать вывод, что даже при экстремальной потере данных из-за наличия большого числа сбойных участков оставшиеся „чистые“ сегменты, объединенные в один сигнал, могут использоваться для приемлемой диагностики режима динамики.

$6^{*}$ Письма в ЖТФ, 2018, том 44, вып. 14 


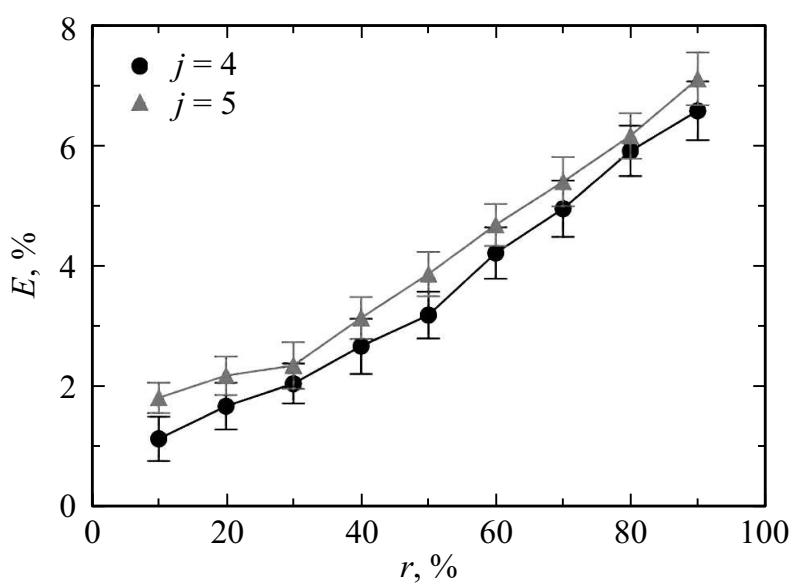

Рис. 2. Зависимости относительной погрешности от объема удаленных данных для двух масштабов наблюдения. Расчеты проведены для вейвлета Добеши $D^{8}$.

В частности, во многих физиологических исследованиях переход от нормы к патологии сопровождается существенно более выраженными изменениями характеристик, превышающими $20-30 \%$ [20], тогда как их изменение в пределах нескольких процентов соответствует вариабельности, связанной с конечной длительностью исследуемых процессов. Аналогичные выводы были сделаны для разных примеров сигналов скорости церебрального кровотока (различные состояния, сосуды и т. д.), а также других вариантов сигналов (динамики сердечного ритма, записей артериального давления крови и т.д.). Процессы физиологической природы в данном случае были выбраны в связи с их сложностью по сравнению с тестовыми примерами, рассмотренными в работах $[17,18]$. Для всех этих сигналов было характерно наличие степенных корреляций, соответствующих нормальной физиологической регуляции.

Таким образом, проведенные исследования позволяют сделать вывод, что при анализе коррелированной динамики сложных процессов режим функционирования исследуемой системы может быть правильно охарактеризован даже при условии экстремальной потери данных при удалении большого количества сбойных участков.

Исследование выполнено при поддержке гранта Российского научного фонда (проект № 17-75-20069).

Письма в ЖТФ, 2018, том 44, вып. 14 


\section{Список литературы}

[1] Bendat J.S., Piersol A.G. Random data: analysis and measurement procedures. 4th ed. N. J.: Wiley, 2010. 640 p.

[2] Urigüen J.A., Garcia-Zapirain B. // J. Neural. Eng. 2015. V. 12. N 3. P. 031001.

[3] Короновский А.А., Макаров В.А., Павлов А.Н., Ситникова Е.Ю., Храмов A.E. Вейвлеты в нейродинамике и нейрофизиологии. М.: Физматлит, 2013. $271 \mathrm{p}$.

[4] Daubechies I. Ten lectures on wavelets. Philadelphia: S.I.A.M., 1992. 378 p.

[5] Дремин И.М., Иванов О.В., Нечитайло В.А. // УФН. 2001. Т. 171. № 5. C. $465-501$.

[6] Addison P.S. The illustrated wavelet transform handbook: introductory theory and applications in science, engineering, medicine and finance. 2nd ed. Boca Raton: CRC Press, 2016. 446 p.

[7] Loizou P.C. Speech enhancement: theory and practice. 2nd ed. Boca Raton: CRC Press, 2013. 705 p.

[8] Selesnick I.W., Baraniuk R.G., Kingsbury N.G. // IEEE Signal Process. Mag. 2005. V. 22. N 6. P. 123-151.

[9] Гусев В.А., Короновский А.А., Храмов А.Е. // Письма в ЖТФ. 2003. Т. 29. B. 18 . C. $61-69$.

[10] Божсокин С.В. // ЖТФ. 2012. Т. 82. В. 7. С. 8-13.

[11] Божокин С.В., Суслова И.М. // ЖТФ. 2013. Т. 83. В. 12. С. 26-32.

[12] Грубов В.В., Руннова А.Е., Короновский А.А., Храмов А.Е. // Письма в ЖТФ. 2017. Т. 43. В. 13. С. 58-64.

[13] Ясин А.С., Павлов А.Н. // Письма в ЖТФ. 2015. Т. 41. В. 14. С. 33-38.

[14] Ясин А.С., Павлова О.Н., Павлов А.Н. // Письма в ЖТФ. 2016. Т. 42. В. 2. C. $50-56$.

[15] Ясин А.С., Павлова О.Н., Павлов А.Н. // Письма в ЖТФ. 2016. Т. 42. В. 16. C. $72-78$.

[16] Ясин А.С., Павлова О.Н., Павлов А.Н. // Письма в ЖТФ. 2017. Т. 43. В. 14. C. $10-18$.

[17] Ma Q.D.Y., Bartsch R.P., Bernaola-Galván P., Yoneyama M., Ivanov P.Ch. // Phys. Rev. E. 2010. V. 81. N 3. P. 031101.

[18] Pavlov A.N., Pavlova O.N., Abdurashitov A.S., Sindeeva O.A., SemyachkinaGlushkovskaya O.V., Kurths J. // Chaos. 2018. V. 28. N 1. P. 013124.

[19] Thurner S., Feurstein M.C., Teich M.C. // Phys. Rev. Lett. 1998. V. 80. N 7. P. $1544-1547$.

[20] Semyachkina-Glushkovskaya O., Pavlov A., Kurths J., Borisova E., Gisbrecht A., Sindeeva O., Abdurashitov A., Shirokov A., Navolokin N., Zinchenko E., Gekalyuk A., Ulanova M., Zhu D., Luo Q., Tuchin V. // Biomed. Opt. Exp. 2015. V. 6. N 10. P. 4088-4097.

Письма в ЖТФ, 2018, том 44, вып. 14 